\title{
Indicateurs de qualité dans le domaine ambulatoire
}

\author{
Maria Wertlia, Sima Nuschin Djalalib, Brigitte Zirbs Savignyc, Adrian Rohrbasserd, Joël Lehmanne, \\ Marc Michael Jungic, Mirjam Rodella Sapiac, Omar Kherad ${ }^{\mathrm{f}}$, Markus Schneemann ${ }^{\mathrm{b}}$, Silvana K. Rampini ${ }^{\mathrm{b}}$, \\ Lars Clarfeld ${ }^{g}$, Jacques Donzéf ${ }^{\text {, Regula Capaul }}{ }^{\text {h }}$
}

a Prof. Dr méd., présidente de la Commission de qualité de la Société Suisse de Médecine Interne Générale SSMIG; ${ }^{b}$ PD Dr méd., membre de la Commission de qualité de la Société Suisse de Médecine Interne Générale SSMIG; ${ }^{\circ}$ Dr méd., membre de la Commission de qualité de la Société Suisse de Médecine Interne Générale SSMIG; ${ }^{d}$ Dr. phil., membre de la Commission de qualité de la Société Suisse de Médecine Interne Générale SSMIG; ${ }^{e}$ M.A., membre de la

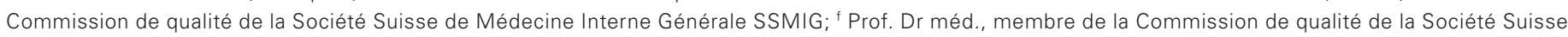
de Médecine Interne Générale SSMIG; ${ }^{9}$ Dr méd., secrétaire général de la Société Suisse de Médecine Interne Générale SSMIG; ${ }^{\mathrm{h}}$ Dr méd., co-présidente de la Société Suisse de Médecine Interne Générale SSMIG, membre de la Commission de qualité de la SSMIG

La Commission de qualité de la Société Suisse de Médecine Interne Générale (SSMIG) publie pour la première fois une liste de six recommandations pour la médecine ambulatoire. L’objectif est d'améliorer la qualité médicale à long terme.

La qualité en médecine s'est récemment invitée dans l'agenda politique de la santé. On enregistre une demande croissante visant à mesurer la qualité des traitements médicaux et à la rendre visible [1]. Depuis de nombreuses années, la Société Suisse de Médecine Interne Générale (SSMIG) s'engage pour une prise en charge de qualité des patients de plus en plus polymorbides - une préoccupation centrale de la médecine in-

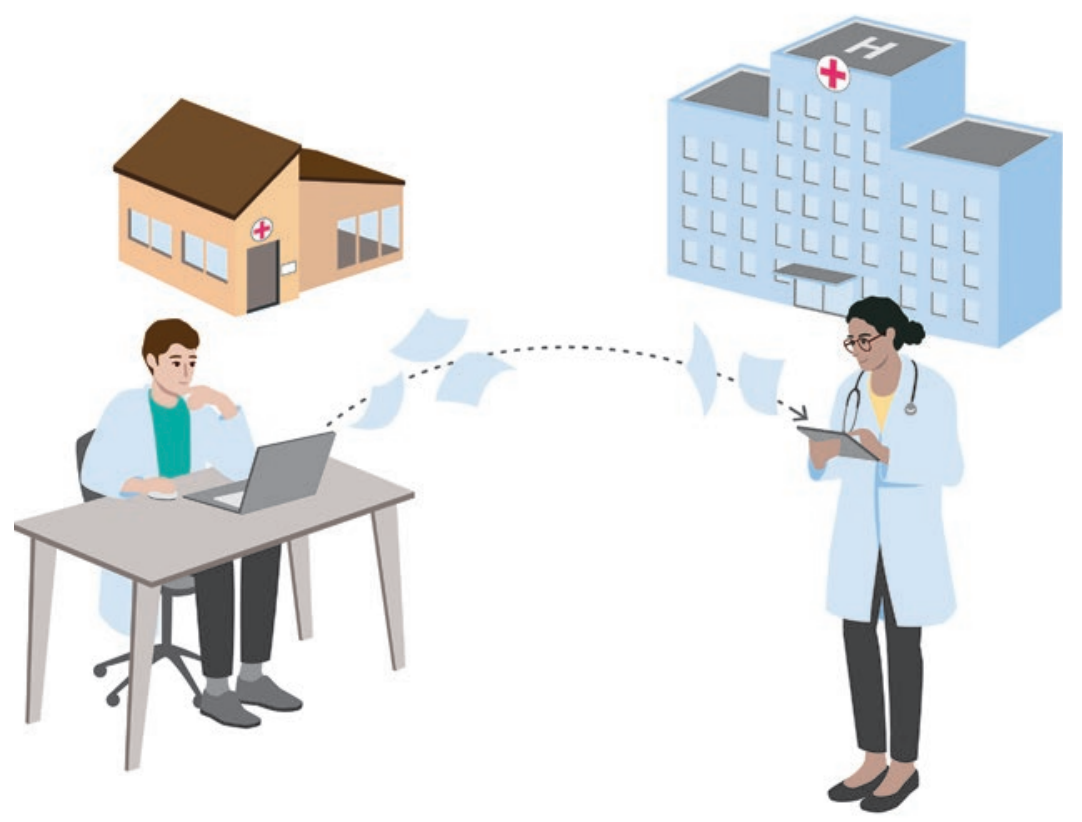

Transmission d'informations médicales entre le médecin généraliste et les spécialistes (hospitaliers). terne générale (MIG) [2]. La Commission de qualité de la SSMIG s'est intéressée de manière approfondie au thème de la mesure de la qualité et a publié pour la première fois en juin 2021 six indicateurs qualité destinés à la médecine stationnaire, qui peuvent être utiles dans la pratique clinique [3]. La SSMIG a maintenant publié six recommandations pour la médecine ambulatoire. Ces indicateurs ont pour objectif de renforcer des mesures d'amélioration de la qualité dans le cadre d'un processus structuré [4].

La qualité médicale est souvent décrite selon trois dimensions [5]: qualité structurelle, qualité des processus et qualité des résultats. Les indicateurs recommandés par la SSMIG s'intéressent à la qualité des soins centrés sur les besoins du patient. Dans ce contexte, les trois premiers indicateurs abordent les questions des soins inappropriés et de surmédicalisation faisant écho à la campagne "smarter medicine» qui peuvent être facilement mis en œuvre dans le quotidien du cabinet et accroître la sécurité des patients.

1. La proportion de patients pour lesquels le médecin de famille envoie un rapport de transfert en cas d'hospitalisation ou de consultation chez un spécialiste. Cet indicateur a pour but d'améliorer la transmission des informations médicales entre le médecin de famille et le spécialiste et/ou l'hôpital. Une transmission efficace des informations, comprenant des questions adressées aux spécialistes ainsi que les principaux résultats médicaux, devrait améliorer la qualité de l'évaluation du patient par le spécialiste et la continuité des soins. La qualité du trai- 


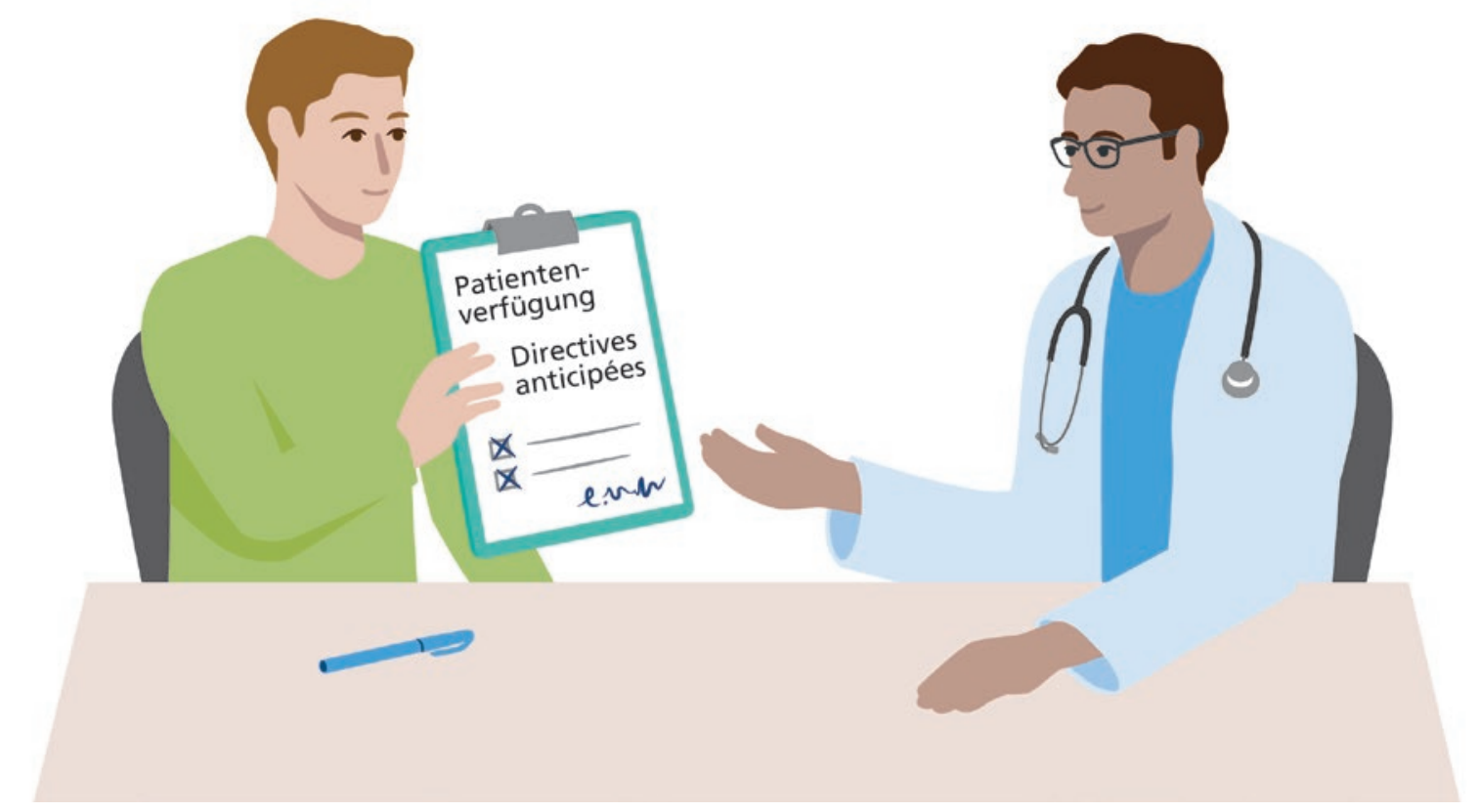

Saisir les préférences du patient dans des directives anticipées.

tement peut ainsi être améliorée, notamment en réduisant la répétition d'examens techniques et de tests de laboratoire.

2. Proportion de patients qui disposent de directives anticipées dans leur dossier médical. Cet indicateur a pour but d'encourager les médecins de famille à aborder de manière proactive et à documenter les besoins et attentes individuels des patients en matière de soins de fin de vie ou lors de la perte des capacités de discernement.

3. Proportion de patients âgés de 65 ans et plus prenant au moins 5 médicaments au long cours et ayant effectué une réconciliation médicamenteuse avec vérification des interactions au cours des 12 derniers mois. Cet indicateur a pour but de détecter et prévenir les interactions médicamenteuses, les effets secondaires potentiels et de réduire la consommation de médicaments inutiles.

4. Proportion de patients âgés de 65 ans et plus interrogés sur la fréquence (nombre) et le mode (déroulement) de chutes éventuellement survenues au cours des 12 derniers mois. Cet indicateur a pour but d'identifier les patients présentant un risque accru de chute, chez lesquels une intervention préventive s'avère judicieuse.

5. Proportion de patients dont le comportement addictif (consommation de nicotine, d'alcool, de drogues, de médicaments) a été abordé par le médecin de famille. Cet indicateur a pour but d'identifier les patients présentant un comportement addictif potentiellement problématique et de mettre en place des stratégies de traitement appropriées.
6. Proportion de patients pour lesquels la prévention et les facteurs liés au mode de vie (p. ex. sédentarité, alimentation, obésité, mesures de prévention adaptées à l'âge) ont été abordés par le médecin de famille. Cet indicateur a pour but d'identifier les patients susceptibles de modifier leur mode de vie afin d'améliorer leur pronostic de santé.

\section{La Commission de qualité de la SSMIG s'est intéressée de manière approfondie au thème de la mesure de la qualité.}

Une description détaillée des indicateurs, la littérature spécialisée et de plus amples informations sont disponibles sur le site Internet de la SSMIG (www.sgaim.ch/ fr/programme-liste-des-complications/qualite-en. html). Dans un second temps, la Commission de qualité de la SSMIG a l'intention d'élaborer des supports à l'attention des médecins concernant ces indicateurs qui soutiennent et guident les processus d'amélioration de la qualité dans les cabinets ambulatoires. Ces documents seront gratuitement mis à la disposition des membres de la SSMIG.

\section{Processus de création de la liste}

Une analyse de la littérature a permis d'identifier au total 36 indicateurs de processus potentiellement appropriés, indicateurs de résultats et 4 indicateurs de qualité structurelle. La qualité structurelle inclut p. ex. le nombre de soignants et leurs qualifications ainsi que la maintenance adéquate des appareils techniques. La 
Tableau 1: Evaluation des indicateurs selon les critères de I'ACP. Les indicateurs ont été jugés pertinents s'ils ont reçu une note moyenne de $\geq 7.0$ dans au moins quatre domaines.

\begin{tabular}{|c|c|c|c|c|c|}
\hline Indicateur & $\begin{array}{l}\text { Importance } \\
\text { clinique }\end{array}$ & Pertinence & $\begin{array}{l}\text { Basé sur des } \\
\text { preuves }\end{array}$ & Clarté & Applicabilité \\
\hline $\begin{array}{l}\text { 1. Proportion de rapports de } \\
\text { transfert }\end{array}$ & 8.5 & 8.0 & 8.5 & 8.0 & 7.0 \\
\hline $\begin{array}{l}\text { 2. Proportion de directives } \\
\text { anticipées }\end{array}$ & 8.0 & 8.0 & 7.0 & 8.0 & 7.0 \\
\hline $\begin{array}{l}\text { 3. Proportion de réconciliation } \\
\text { médicamenteuse }\end{array}$ & 9.0 & 9.0 & 8.0 & 8.0 & 7.0 \\
\hline $\begin{array}{l}\text { 5. Proportion de comportements } \\
\text { addictifs identifiés }\end{array}$ & 8.0 & 7.0 & 7.0 & 7.0 & 7.0 \\
\hline 6. Proportion de prévention & 7.0 & 7.0 & 7.5 & 7.0 & 6.0 \\
\hline
\end{tabular}

qualité des processus comprend p. ex. le traitement d'une maladie selon l'état actuel des connaissances. La qualité des résultats mesure p. ex. le taux de guérison, les réhospitalisations non prévues, la satisfaction des patients ou la mortalité.

Lors d'une retraite en février 2020, les indicateurs ont été analysés dans le cadre d'ateliers. Six indicateurs stationnaires et six indicateurs ambulatoires ont été jugés pertinents. Pour la commission, il était important que les indicateurs soient utilisables au quotidien, afin d'encourager et soutenir un cycle d'amélioration de la qualité en médecine interne générale.
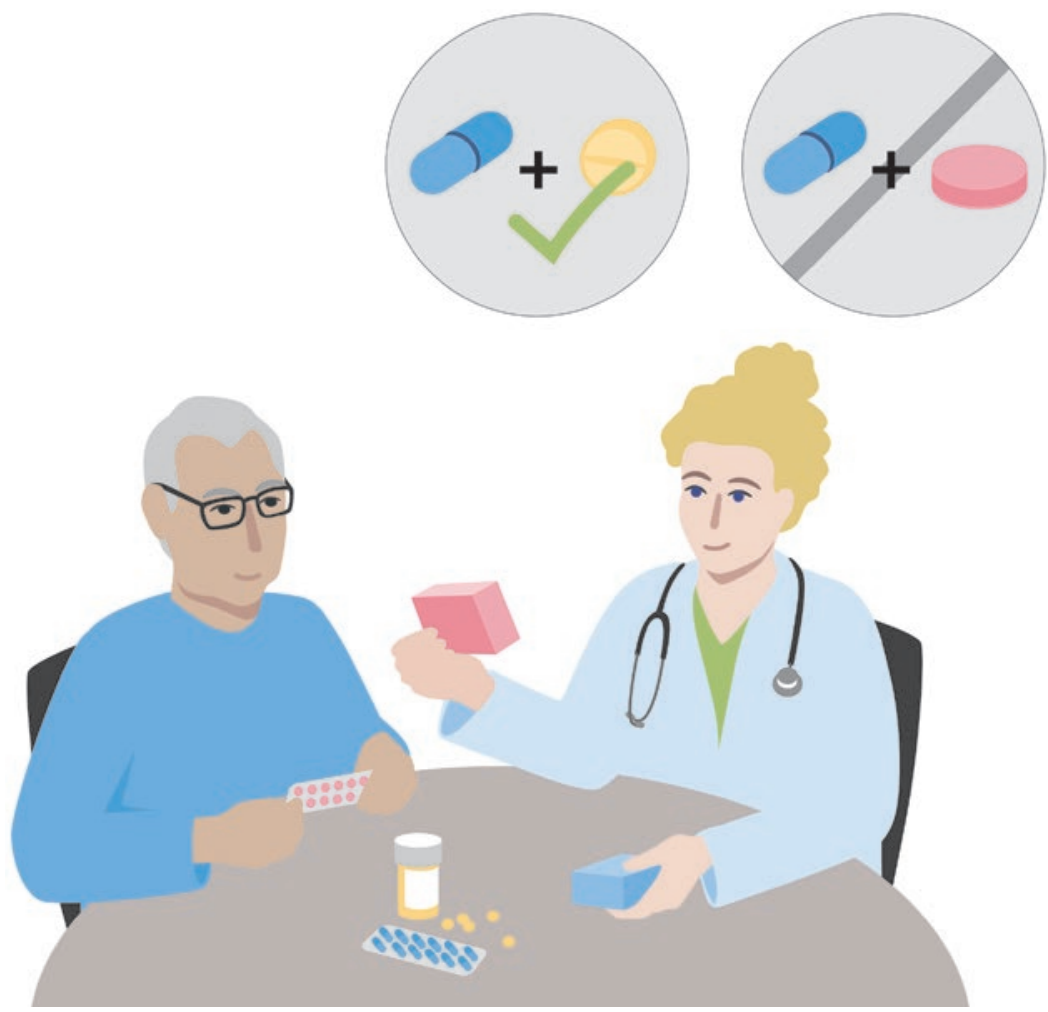

Identifier les interactions médicamenteuses et prévenir les effets secondaires.
Ont été ainsi identifiés en premier lieu des indicateurs de processus. La commission a décidé de ne pas utiliser d'indicateurs de résultats, car leur saisie est très complexe et n'est généralement pertinente qu'en combinaison avec des indicateurs de processus. Les indicateurs

\section{Des supports visant à soutenir et à guider les} processus d'amélioration de la qualité dans les cabinets ambulatoires seront élaborés.

sélectionnés ont été formulés, et leur applicabilité ainsi que leur mesurabilité ont été optimisées. Tous les indicateurs ont été vérifiés selon les critères appliqués par l'American College of Physicians (ACP) [6]:

1. Importance: l'utilisation de l'indicateur aura pour conséquence une amélioration mesurable et significative des critères cliniques (impact important, écart de performance).

2. Traitement approprié: éviter la surabondance ou l'usage inapproprié de prestations médicales.

3. Preuve clinique de haute qualité: des études ont révélé qu'une amélioration de la qualité permet également d'améliorer le traitement.

4. Validité et fiabilité: l'indicateur permet de détecter et mesurer de manière reproductible l'événement souhaité.

5. Applicabilité: peut être influencée par les médecins, est applicable (clarté, charge de travail).

L'évaluation de l'ACP est présentée dans le tableau 1. Lorsque cela s'est avéré nécessaire, les indicateurs ont été optimisés à nouveau après une révision interne. Cette étape a été suivie d'une révision externe par divers experts.

\section{Défi relatif à la mise en œuvre}

L’objectif de la commission de qualité était d'identifier, sur la base de la littérature spécialisée et de l'impor- 


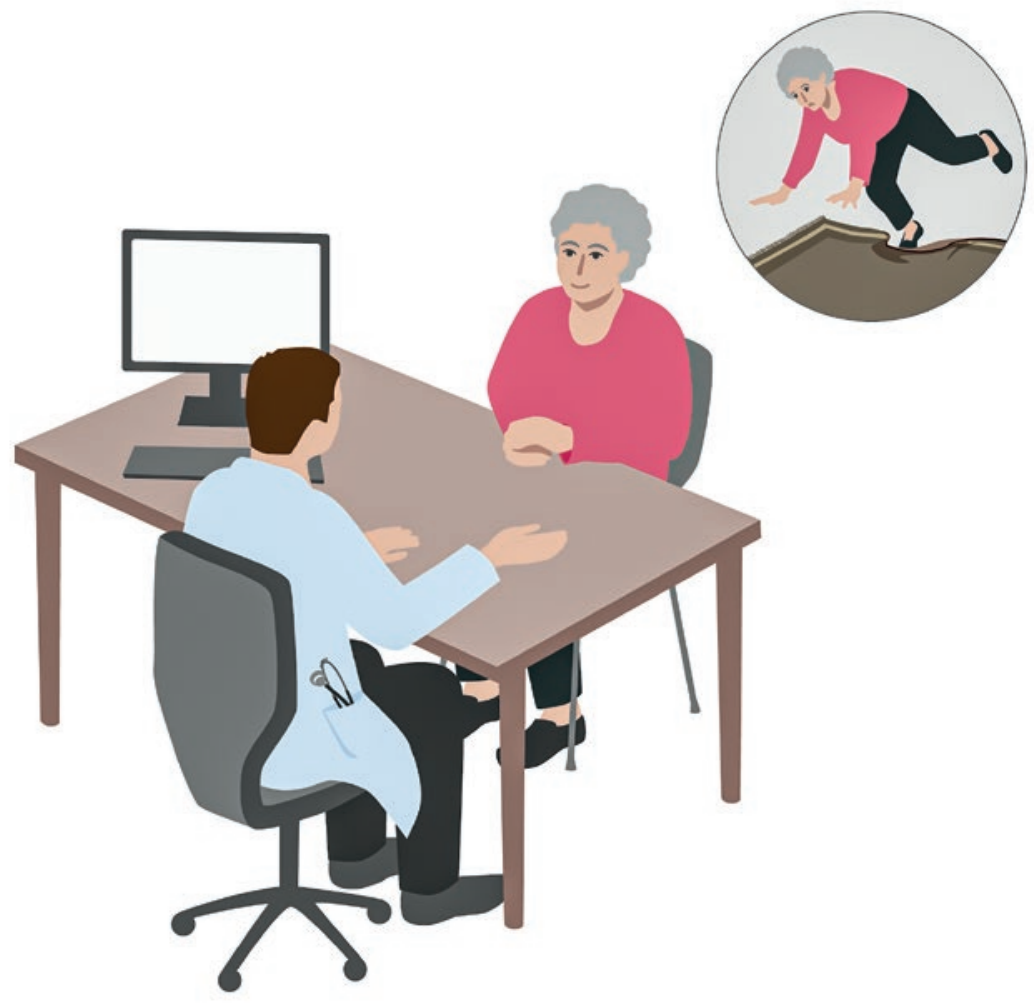

Reconnaître les risques de chute et leur prévention.

Prof. Dr méd. et phil. Maria Wertli

Présidente de la Commission de qualité de la SSMIG Clinique universitaire de médecine interne générale Hôpital de l’lle, Berne Freiburgstrasse 16p CH-3010 Berne tance clinique, des indicateurs susceptibles de soutenir les processus d'amélioration de la qualité en MIG. Toutefois, la condition de base est la mise en place de méthodes de mesures fiables. Pour pouvoir saisir un grand nombre d'indicateurs, les dossiers médicaux informatisés doivent être adaptés afin de pouvoir collecter de manière systématique et uniforme les résultats. Il est par ailleurs impératif que les ressources humaines et financières nécessaires à cette fin soient prises en compte dans le système de rémunération.

\section{Remerciements}

Nous remercions tous les collègues et experts pour leur examen critique et leurs suggestions: Dr méd. Philippe Luchsinger (président de mfe), Prof. Dr méd. Stefan Neuner-Jehle (représentant de l'Institut de médecine de famille de l'Université de Zurich) et Prof. Dr méd. Sven Streit (Institut de médecine de famille de l'Université de Berne, BIHAM et membre de la Commission nationale pour la qualité). Nous remercions également Ursula Käser (responsable du service qualité, formation postgraduée et continue de la SSMIG) pour son excellent travail et son soutien.

\section{Crédits photos}

SSMIG (illustrations: Hahn+Zimmermann)

\section{Références}

1 Le rapport national sur la qualité indique un grand potentiel d'amélioration du système de santé suisse, OFSP, 8.11.2019.

2 smarter medicine. Weitere Top-5-Liste für die ambulante Allgemeine Innere Medizin. Schweiz Ärzteztg. 2021;102(17):572-3.

3 Wertli M. Indicateurs de qualité dans le domaine stationnaire. Bull Med Suisses. 2021;102(26):877-80.

4 Relevé, analyse et publication de données concernant la qualité des traitements médicaux. Bull Med Suisses. 2009;90(26):1044-54.

5 Donabedian A. Evaluating the Quality of Medical Care. The Milbank Quarterly. 2005;83(4):691-729.

6 MacLean CH. Time Out - Charting a Path for Improving Performance Measurement. NEJM. 2018;378:1757-61.

Lisez aussi l'entretien de la Dre méd. Regula Capaul, co-présidente et membre de la Commission de qualité de la SSMIG, sur le site du Bulletin des médecins suisses: www.bullmed. ch $\rightarrow$ tour d'horizon.

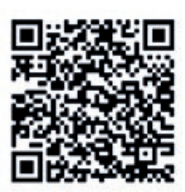

\section{L'essentiel en bref}

- La SSGIM a formulé six indicateurs de qualité pour la médecine ambulatoire.

- Les indicateurs recommandés visent à améliorer les processus de qualité de la prise en charge du patient.

- Les indicateurs sélectionnés ont été examinés selon les critères d'importance, de traitement approprié, de preuves cliniques de haute qualité, de validité et de fiabilité ainsi que d'applicabilité. 